\title{
CRESCIMENTO VEGETATIVO DE MACIEIRAS 'FUJI SUPREMA' SOB INFLUÊNCIA DA ÉPOCA DE APLICAÇÃO DE PROEXADIONE CÁLCIO'
}

\author{
FERNANDO JOSÉ HAWERROTH² \& JOSÉ LUIZ PETRI ${ }^{3}$
}

RESUMO- O uso de reguladores de crescimento que atuam na biossíntese de giberelinas, como o proexadione cálcio, pode possibilitar adequado balanço entre o desenvolvimento vegetativo e o reprodutivo, fundamental para a maximização dos índices produtivos. No entanto, a resposta ao uso deste regulador de crescimento depende do padrão de crescimento dos ramos. Objetivou-se com este trabalho avaliar o efeito de diferentes épocas de aplicação de proexadione cálcio no controle do desenvolvimento vegetativo de macieiras 'Fuji Suprema', sob condições climáticas do Sul do Brasil. Avaliaram-se diferentes épocas de aplicação de proexadione cálcio, a $330 \mathrm{~g} \mathrm{ha}^{-1}$, em dois ciclos produtivos, utilizando o delineamento experimental em blocos casualizados, com dez repetições compostas por uma planta. No ciclo de 2008/2009, foram avaliadas seis épocas de aplicação $(28 ; 58 ; 88 ; 118 ; 148$ e 178 dias após a plena floração - DAPF). No ciclo de 2009/2010, foram avaliadas sete épocas $(20 ; 50 ; 80 ; 110 ; 140 ; 170$ e 200 DAPF), ambas comparadas com o tratamentotestemunha(sem aplicação). O proexadione cálcio em uma única aplicação, a $330 \mathrm{~g} \mathrm{ha}^{-1}$, aplicado até 28 dias após a plena floração, pode reduzir o número, a massa e o comprimento médio dos ramos podados em macieiras 'Fuji Suprema'; porém, sobre condições que favoreçam o demasiado desenvolvimento vegetativo, o efeito mostra-se nulo. O uso de $330 \mathrm{~g} \mathrm{ha}^{-1}$ de proexadione cálcio em uma única aplicação, indiferentemente da época de aplicação, não altera a produção de macieiras 'Fuji Suprema'.

Termos para indexação: Malus domestica, regulador de crescimento, biossíntese de giberelinas, produção de frutos.

\section{VEGETATIVE GROWTH 'FUJI SUPREMA’APPLES UNDER INFLUENCE OF PROHEXADIONE CALCIUM APPLICATION TIMES}

\begin{abstract}
The use of plant growth regulators that act on the biosynthesis of gibberellins, as proexadione calcium, may enable proper balance between vegetative growth and fruiting to maximize production indexes. However, the response of this plant growth regulator depends on the pattern of shoot growth. Thus, the aim of this study was to evaluate the effect of application times of prohexadione calcium on vegetative growth control of apple 'Fuji Suprema', in the climatic conditions of Southern Brazil. It was evaluated different application times of prohexadione calcium at $330 \mathrm{~g} \mathrm{ha}^{-1}$, in two growing seasons, using randomized complete block design with ten replication of a plant. In the 2008/2009 growing season were evaluated six times of application $(28,58,88,118,148$, and 178 DAFB, days after full bloom), and in the 2009/2010 season were evaluated seven times $(20,50,80,110,140,170$, and 200 DAFB), both compared with check treatment (no application). The prohexadione calcium sprayed in a single application at $330 \mathrm{~g} \mathrm{ha}^{-1}$ until 28 days after full bloom may reduce the number, weight and average length of pruned shoots in 'Fuji Suprema' apple trees, but in conditions of excessive vegetative growth have not effect. The use prohexadione calcium at $330 \mathrm{~g} \mathrm{ha}^{-1}$ in a single application, regardless of timing application has not effect on fruit production of 'Fuji Suprema' apples. Index terms: Malus domestica, plant growth regulador, gibberellins biosynthesis, fruit production.
\end{abstract}

${ }^{1}$ (Trabalho 375-13). Recebido em: 03-10-2013. Aceito para publicação em: 14-02-2014.

${ }^{2}$ Eng. Agr., DSc., Pesquisador, Embrapa Agroindústria Tropical, 60511-110, Fortaleza-CE, Brasil. E-mail: fernando.hawerroth@ embrapa.br

${ }^{2}$ Eng. Agr., MSc., Pesquisador, Epagri - Estação Experimental de Caçador, Caçador-SC, Brasil. E-mail: petri@epagri.sc.gov.br. 


\section{INTRODUÇÃO}

Os sistemas de plantio em alta densidade têm sido preconizados na produção de frutíferas de clima temperado, sendo imprescindíveis o controle do vigor e o controle da produção das plantas para adoção de tais sistemas. A obtenção de plantas menores, que entram em produção no segundo ano após o plantio, é um pré-requisito para garantir a regularidade na produção de frutas de alta qualidade, além de proporcionar melhor uso da terra e reduzir os custos na operacionalização da poda e da colheita (MAAS, 2008).

Pomares com plantas vigorosas reduzem a penetração de luz no interior da copa (PRIVÉ et al., 2004), o que acarreta diminuição da produtividade e qualidade dos frutos, aumento do custo de poda, além de dificultar o controle de doenças e pragas pela redução da eficiência de aplicações fitossanitárias (HAWERROTH et al., 2012a). Nas condições climáticas do Sul do Brasil, o período de desenvolvimento vegetativo de macieiras 'Gala' e 'Fuji' mostra-se superior ao observado em típicas regiões de clima temperado, o qual, associado a altas temperaturas e altos índices pluviométricos durante o ciclo, pode resultar em crescimento de ramos acima de $1 \mathrm{~m}$, sobretudo em anos de baixa frutificação. Por esta razão, a utilização de práticas culturais que promovam o adequado balanço entre o desenvolvimento vegetativo e a frutificação da macieira mostra-se fundamental ao aumento da eficiência produtiva e à melhoria da qualidade dos frutos.

O controle do desenvolvimento vegetativo deve ser realizado, preferencialmente, por meios naturais, tais como o uso de porta-enxertos de reduzido vigor, o uso de podas de formação e de frutificação. No entanto, se essas soluções não são passíveis de serem implementadas ou não são suficientes para restringir o vigor da copa, a redução do desenvolvimento vegetativo pode ser obtida pelo uso de reguladores de crescimento.

A redução do crescimento de ramos pode ser obtida pelo uso de substâncias inibidoras da biossíntese de giberelinas. Giberelinas ativas, como a $\mathrm{GA}_{1}$ desempenham um papel importante no alongamento de ramos. Seu precursor imediato é a $\mathrm{GA}_{20}$, que é biologicamente inativa. A conversão de $\mathrm{GA}_{20}$ para $\mathrm{GA}_{1}$ é mediada pela enzima $\mathrm{GA}_{20}{ }^{-}$ $3 \beta$-hidroxilase (RADEMACHER, 2009). O proexadione cálcio regula os estágios finais da biossíntese de giberelinas por interferir na 3 $\beta$-hidroxilação (ILIAS ; RAJAPAKSE, 2005). $\mathrm{O}$ efeito líquido é a redução de giberelinas biologicamente ativas $\left(\mathrm{GA}_{1}\right)$, pelo aumento nos níveis de giberelinas inativas $\left(\mathrm{GA}_{20}\right)$, uma vez que este regulador de crescimento bloqueia a conversão

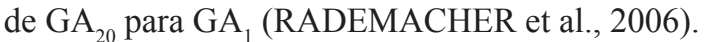

Trabalhos de Miller (2002) mostraram que o proexadione cálcio pode reduzir de 18 a $60 \%$ o crescimento de ramos de macieiras. Resultados obtidos por Hawerroth (2010), sob condições climáticas do Sul do Brasil, mostraram a efetividade do proexadione cálcio na redução do desenvolvimento vegetativo em macieiras 'Imperial Gala' e 'Fuji Suprema', enxertadas no porta-enxerto 'Marubakaido' com interenxerto de M9. Em função de a resposta do proexadione cálcio ser dependente do padrão sazonal de crescimento dos ramos, para aperfeiçoar o uso deste regulador de crescimento no manejo da macieira, faz-se necessária a definição das épocas de aplicação que proporcionem maior eficiência no controle do desenvolvimento vegetativo.

O objetivo desse trabalho foi avaliar o efeito de diferentes épocas de aplicação de proexadione cálcio no controle do desenvolvimento vegetativo de macieiras 'Fuji Suprema', sob condições climáticas do Sul do Brasil.

\section{MATERIAL E MÉTODOS}

Os experimentos foram realizados em pomar comercial localizado no município de Fraiburgo-SC (latitude $27^{\circ} 04^{\prime} \mathrm{S}$, longitude $50^{\circ} 52^{\prime} \mathrm{W}$ ), durante as safras de 2008/2009 e 2009/2010. O clima da região é classificado como $\mathrm{Cfb}$, temperado, constantemente úmido, com verão ameno, segundo classificação de Köppen. Os dados de temperatura média e a precipitação pluvial mensal para o período de execução do experimento estão apresentados na Figura 1.

Foram utilizadas macieiras 'Fuji Suprema' com dez anos de idade, enxertadas sobre o portaenxerto 'Marubakaido', com interenxerto de 'M.9', implantadas na densidade de plantio de 2.500 plantas $\mathrm{ha}^{-1}$, com espaçamento de $4 \mathrm{~m}$ entre linhas e $1 \mathrm{~m}$ entre plantas. Desde a implantação do pomar, as plantas, conduzidas no sistema de condução em líder central, foram manejadas de acordo com as práticas recomendadas no sistema de produção da macieira (SANHUEZA et al., 2006).

Os experimentos consistiram na avaliação de diferentes épocas de aplicação de proexadione cálcio, a $330 \mathrm{~g} \mathrm{ha}^{-1}$, em dois ciclos produtivos. No ciclo de 2008/2009 foram avaliados os seguintes tratamentos: 1) proexadione cálcio $(\mathrm{PCa})$ aos 28 DAPF- dias após a plena floração; 2) PCa aos 58 
DAPF; 3) PCa aos 88 DAPF; 4) PCa aos 118 DAPF; 5) $\mathrm{PCa}$ aos $148 \mathrm{DAPF}$; 6) PCa aos $178 \mathrm{DAPF}$; e 7) testemunha (sem aplicação). No ciclo de produção de 2009/2010, foram avaliados oito tratamentos: 1) $\mathrm{PCa}$ aos $20 \mathrm{DAPF}$; 2) PCa aos $50 \mathrm{DAPF}$; 3) PCa aos 80 DAPF; 4) PCa aos 110 DAPF; 5) PCa aos 140 DAPF; 6) PCa aos 170 DAPF; 7) PCa aos 200 DAPF; e 8) testemunha (sem aplicação).

Como fonte de proexadione cálcio foi utilizado o produto comercial Viviful ${ }^{\circledR}$, contendo $27,5 \%$ de ingrediente ativo. A aplicação dos tratamentos foi realizada por meio de aspersão com pulverizador costal motorizado, equipado com bico Leque DS, utilizando volume médio de calda correspondente a $1.000 \mathrm{~L} \mathrm{ha}^{-1}$.

A produção de frutos por planta foi quantificada em ambos os ciclos avaliados. Ao final do período hibernal de cada ciclo, foi efetuada a poda de ramos, sendo avaliada a massa $\mathrm{kg} /$ planta $^{-1}$ e o número médio de ramos podados por planta (ramos/ planta $^{-1}$ ). $\mathrm{O}$ comprimento dos ramos podados foi mensurado em cada planta, obtendo-se a média e o coeficiente de variação do comprimento de ramos podados de cada tratamento. A partir da mensuração do comprimento dos ramos, estes foram agrupados em quatro categorias: ramos menores que $30 \mathrm{~cm}$; ramos com comprimento maior ou igual a 30 e menores que $60 \mathrm{~cm}$; ramos com comprimento maior ou igual a $60 \mathrm{~cm}$ e menores que $90 \mathrm{~cm}$; e ramos com comprimento maior ou igual a $90 \mathrm{~cm}$.

Em ambos os ciclos de avaliação, o delineamento experimental foi em blocos casualizados, com dez repetições, sendo cada repetição composta por uma planta. Os resultados obtidos foram submetidos à análise da variância, cujas variáveis significativas $(\mathrm{p}<0,05)$ tiveram as médias comparadas pelo teste Tukey, a 5\% de probabilidade de erro. Os procedimentos de análise foram realizados por meio do programa SAS (SAS INSTITUTE INC., 2004).

\section{RESULTADOS E DISCUSSÃO}

No ciclo de 2008/2009, a maior proporção de ramos com comprimento inferior a $30 \mathrm{~cm}$ foi verificado em macieiras 'Fuji Suprema' tratadas com proexadione cálcio aplicado aos $28 \mathrm{DAPF}$, sendo significativamente superior ao observado nas demais épocas de aplicação deste regulador de crescimento e no tratamento-testemunha (Figura 2). Em contrapartida, a aplicação de $\mathrm{PCa}$ aos 28 DAPF determinou a menor proporção de ramos com comprimento entre 30 e $60 \mathrm{~cm}$ quando comparado aos demais tratamentos. A proporção de ramos podados com comprimento entre 60 e $90 \mathrm{~cm}$ e maiores que 90 $\mathrm{cm}$ não foi alterada pelo uso de proexadione cálcio, indiferentemente da época de aplicação (Figura 2).

No ciclo de 2009/2010 não foram observadas diferenças entre o tratamento-testemunha e as diferentes épocas de aplicação de proexadione cálcio quanto à proporção de ramos podados menores que $30 \mathrm{~cm}$ de comprimento (Figura 2). A aplicação de 330 $\mathrm{g} \mathrm{ha}^{-1}$ de proexadione cálcio aos 20 DAPF determinou maior proporção de ramos com comprimento entre 30 e $60 \mathrm{~cm}$ em comparação ao tratamento-testemunha, proexadione cálcio aos $50 \mathrm{DAPF}$ e demais aplicações deste regulador de crescimento a partir dos 140 DAPF. Não houve diferença significativa entre tratamentos quanto à proporção de ramos podados com comprimento entre 60 e $90 \mathrm{~cm}$. Aplicações de proexadione cálcio realizadas entre 20 e 110 DAPF determinaram menor quantidade de ramos com comprimento superior a $90 \mathrm{~cm}$, embora ambos não tenham diferido de plantas não tratadas com este regulador de crescimento (Figura 2).

A exceção da aplicação de proexadione cálcio aos 28 DAPF, no ciclo de 2008/2009, a maior proporção de ramos podados apresentava comprimento entre 30 e $60 \mathrm{~cm}$ em ambos os ciclos avaliados. De maneira geral, em ambos os ciclos avaliados, as aplicações de proexadione cálcio realizadas entre 20 e 28 DAPF aumentaram a proporção de ramos nas classes de menor comprimento, reduzindo o número de ramos nas classes de maior tamanho. Tal resposta evidencia que o proexadione cálcio, em uma única aplicação, tem efeito no controle do desenvolvimento vegetativo quando aplicado no início do período de desenvolvimento vegetativo. Segundo Rademacher (2009), o controle do crescimento vegetativo mostra-se mais efetivo quando o crescimento dos ramos está sob controle desde o início do período de crescimento, em que a época de máxima eficiência do proexadione cálcio muitas vezes corresponde ao final do florescimento.

A aplicação de proexadione cálcio aos 28 DAPF repercutiu na redução do número de ramos podados em macieiras 'Fuji Suprema' no ciclo de 2008/2009, quando comparada ao tratamentotestemunha e demais épocas de aplicação deste regulador de crescimento (Figura 3). A mesma resposta foi verificada para a massa total de ramos podados e para o comprimento médio dos ramos podados. Em comparação a plantas do tratamentotestemunha, o uso de proexadione cálcio aos 28 DAPF repercutiu na diminuição de $26,5 \%$ tanto no número de ramos podados quanto no comprimento médio dos ramos. A redução do número e do 
comprimento médio dos ramos podados em plantas tratadas com proexadione cálcio aos $28 \mathrm{DAPF}$ proporcionou a redução de $54,7 \%$ da massa de ramos podados em relação a plantas não tratadas com proexadione cálcio. A redução do número, massa e comprimento dos ramos podados, em resposta à aplicação de proexadione cálcio, indica que esta substância minimiza a formação de fortes drenos vegetativos, reduzindo o desenvolvimento excessivo do dossel e, consequentemente, reduzindo a necessidade de poda hibernal.

No ciclo de 2009/2010, o uso de proexadione cálcio, indiferentemente da época de aplicação, não alterou o número e a massa total de ramos podados e o comprimento médio dos ramos podados em relação ao tratamento-testemunha. De acordo com Privé et al. (2006), a resposta ao uso de proexadione cálcio depende do padrão sazonal do crescimento da parte aérea, a qual é influenciada pela cultivar, porta-enxerto, manejo das plantas e por fatores ambientais, como a temperatura e a precipitação. A resposta diferenciada ao uso de proexadione cálcio no ciclo de 2009/2010 em relação ao ciclo anterior pode ser justificada pela maior precipitação pluvial e temperatura média mais elevada no período vegetativo, condições climáticas mais favoráveis ao maior desenvolvimento vegetativo no ciclo de 2009/2010. Assim, pode-se inferir que, em condições favoráveis ao maior desenvolvimento vegetativo é necessária a realização de mais de uma aplicação por ciclo.

De acordo com Miller (2002) e Bubán et al. (2004), a efetividade do proexadione cálcio no número de ramos podados é maior quando este fitorregulador é aplicado em menores concentrações e em múltiplas aplicações ao longo do ciclo, em comparação ao uso de uma única aplicação em maior concentração. Em macieiras 'Fuji Suprema', enxertadas sobre Marubakaido com interenxerto de M.9, Hawerroth (2010) verificou que aplicações parceladas de proexadione cálcio, 165 a $660 \mathrm{~g}$ $\mathrm{ha}^{-1}$, reduziram significativamente a massa total e o comprimento de ramos podados sob condições climáticas do Sul do Brasil.

Em razão da degradação relativamente rápida de proexadione cálcio (RADEMACHER; KOBER, 2003), o aumento do número de aplicações poderia intensificar o controle do desenvolvimento vegetativo (ELFVING et al., 2003). Para Hawerroth et al. (2012a), pode ser indicada a aplicação adicional de prohexadione cálcio ou a realização de poda-verde conforme o crescimento dos ramos, para reduzir o tamanho final dos ramos e aumentar a proporção de ramos produtivos para os ciclos de produção posteriores.

Em ambos os ciclos avaliados, não foram observadas diferenças entre épocas de aplicação de proexadione cálcio e o tratamento-testemunha quanto à variabilidade do comprimento dos ramos podados.

A produção por planta não foi afetada pelo uso de proexadione cálcio, indiferentemente da época de aplicação efetuada em macieiras 'Fuji Suprema'. Hawerroth et al. (2012b), ao avaliarem épocas de aplicação de proexadione cálcio em macieiras 'Imperial Gala', não observaram alterações na produção e na massa fresca dos frutos pelo uso desse fitorregulador. Da mesma forma, Medjdoub et al. (2004) não evidenciaram efeito da aplicação de proexadione cálcio na eficiência produtiva de macieiras 'Smoothee Golden Delicious'. De acordo com Basak e Krzewiñska (2006), apesar de o proexadione cálcio restringir o crescimento de ramos, este não afeta a produção de frutos, embora, em alguns casos, exista tendência de aumento de produtividade após a aplicação deste inibidor de biossíntese de giberelinas. Em contrapartida, Petri et al. (2011) observaram aumento da produção de frutos de macieiras Gala e Fuji, enxertadas sobre o porta-enxerto M.7, pela aplicação de proexadione cálcio, além de diminuir o crescimento de ramos. Segundo estes autores, quando aplicados doses variando de 160 a $640 \mathrm{~g} \mathrm{ha}^{-1}$ aos 20 DAPF e repetindo aos 50 DAPF, o aumento da produção em relação ao tratamento-testemunha foi de 18,0 a $41,6 \%$ em macieiras 'Gala', e de 4,8 a 52,4\% em macieiras 'Fuji'.

A ausência de efeito da aplicação de proexadione cálcio na produção de frutos por planta parece estar relacionada à limitada efetividade dos tratamentos testados no controle do desenvolvimento vegetativo de macieiras 'Fuji Suprema'. Conforme abordado por Hawerroth et al. (2012a), a resposta do proexadione cálcio na produção por planta depende do equilíbrio do desenvolvimento vegetativoprodutivo proporcionada pelo uso deste regulador de crescimento, em que a restrição do desenvolvimento vegetativo aumenta a capacidade produtiva das plantas pela maior disponibilidade de fotoassimilados aos frutos. 


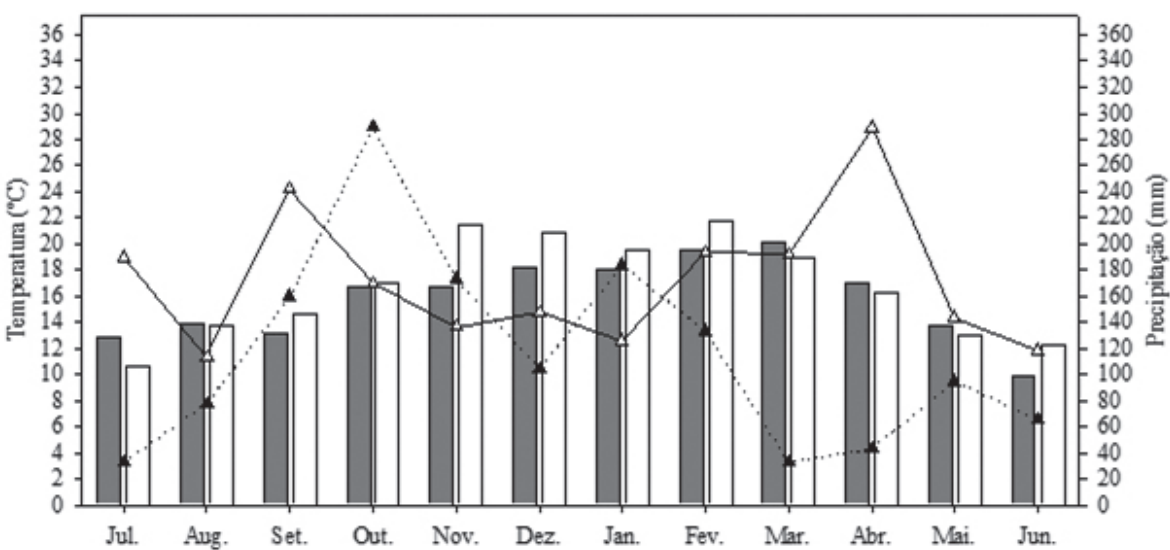
$\square$ Temperatura média mensal no ciclo 2008-2009 $\quad$...... Precipitação mensal no ciclo 2008-2009
$\square$ Temperatura média mensal no ciclo 2009-2010 $\quad$ - Precipitação mensal no ciclo 2009-2010

FIGURA 1-Temperatura média mensal e precipitação pluvial mensal para os meses de julho a junho, nos ciclos de 2008/2009 e 2009/2010. Fraiburgo-SC.

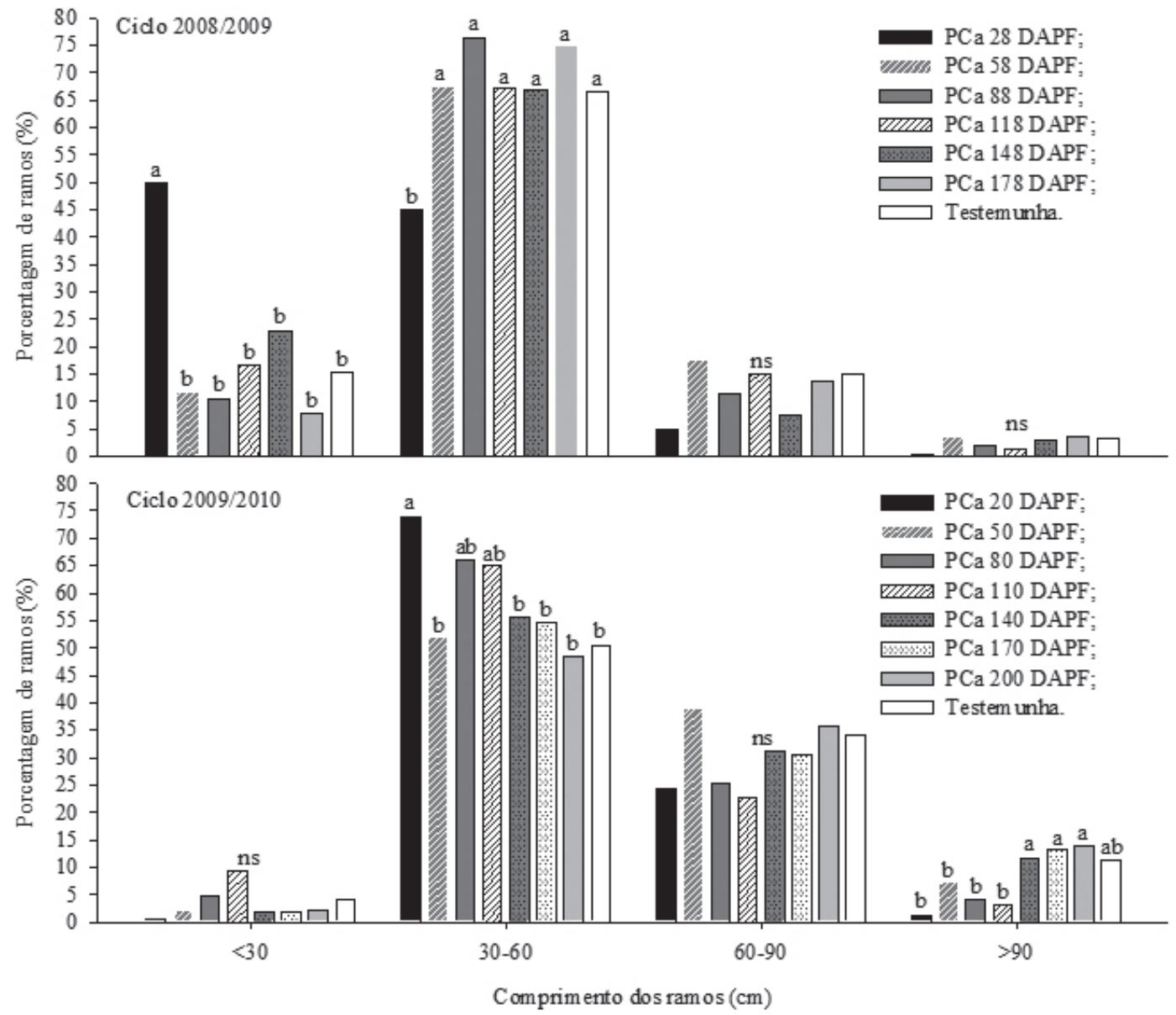

FIGURA 2- Porcentagem de ramos em classes de acordo com o comprimento dos ramos em macieiras 'Fuji Suprema', em função da época de aplicação de proexadione cálcio (PCa) a $330 \mathrm{~g} \mathrm{ha}^{-1}$, nos ciclos de 2008-2009 e 2009-2010. Fraiburgo-SC. DAPF - dias após a plena floração; ns - não significativo pelo teste $\mathrm{F}(\mathrm{p}<0,05)$; Médias seguidas de mesmas letras minúsculas, em cada classe de comprimento de ramos, não diferem significativamente, pelo teste de Tukey $(p<0,05)$. 

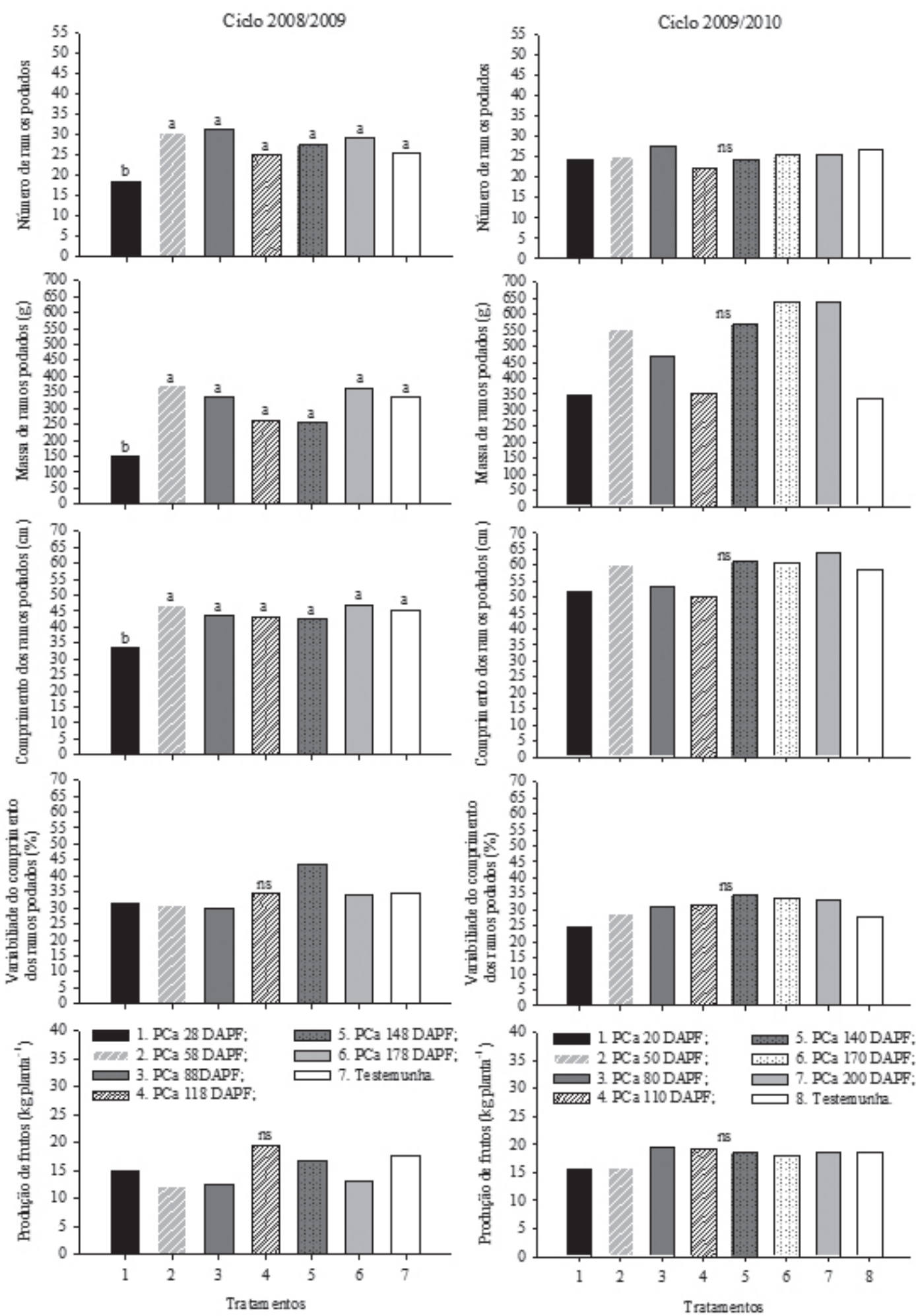

FIGURA 3- Efeito da época de aplicação de proexadione cálcio (PCa) a $330 \mathrm{~g} \mathrm{ha}^{-1}$ sobre o número, massa e comprimento médio de ramos podados, a variabilidade do comprimento de ramos podados e a produção de macieiras 'Fuji Suprema', nos ciclos de 2008/2009 e 2009/2010. Fraiburgo-SC. DAPF - dias após a plena floração; ns - não significativo pelo teste $\mathrm{F}(\mathrm{p}<0,05)$; Médias seguidas de mesmas letras minúsculas não diferem significativamente, pelo teste de Tukey $(\mathrm{p}<0,05)$. 


\section{CONCLUSÕES}

O proexadione cálcio, em uma única aplicação, a $330 \mathrm{~g} \mathrm{ha}^{-1}$, aplicado até 28 dias após a plena floração, pode reduzir o número, a massa e o comprimento médio dos ramos podados em macieiras 'Fuji Suprema'; porém, sob condições que favoreçam o demasiado desenvolvimento vegetativo, o efeito mostra-se nulo.

O uso de $330 \mathrm{~g} \mathrm{ha}^{-1}$ de proexadione cálcio em uma única aplicação, indiferentemente da época de aplicação, não altera a produção de macieiras 'Fuji Suprema'.

\section{REFERÊNCIAS}

BASAK, A.; KRZEWIÑSKA, D. Effect of prohexadione-ca (Regalis ${ }^{\circledR}$ ) on the effectiveness of NAA and BA used for fruitlet thinning in apple trees. Acta Horticulturae, The Hague, v. 727, p. 139-144, 2006.

BUBÁN, T.; CSIZÁR, L.; SALLAI, P.; VARGA, A. Experiences with the bioregulator prohexadione-Ca used in apple and pear orchards. Acta Horticulturae, The Hague v. 636, p. 67-74, 2004.

ELFVING, D.C.; LOMBARDINI, L.; MCFERSON, J.R.; DRAKE, S.R.; FAUBION, D.F.; AUVIL, T.D.; VAN EE, G.; VISSER, D.B. Effects of directed applications of prohexadione-calcium to tops of mature pear trees on shoot growth, light penetration, pruning and fruit quality. Journal of the American Pomological Society, University Park, v. 57, p. 4557, 2003.

HAWERROTH, F.J. Uso de fitorreguladores para controle do desenvolvimento vegetativo e aumento da frutificação em macieira e pereira. 2010. 154 f. Tese (Doutorado em Agronomia - Fruticultura de Clima Temperado), Faculdade de Agronomia 'Eliseu Maciel', Universidade Federal de Pelotas, Pelotas. 2010.

HAWERROTH, F.J.; PETRI, J.L.; FACHINELLO, J.C.; HERTER, F.G.; PREZOTTO, M.E.; HAAS, L.B.; PRETTO, A. Redução da poda hibernal e aumento da produção de pereiras 'Hosui' pelo uso de prohexadiona cálcio. Pesquisa Agropecuária Brasileira, Brasília, v. 47, p. 939-947, 2012a.
HAWERROTH, F.J.; PETRI, J.L.; LEITE, G.B.; YOSHIKAWA, E.R. Épocas de aplicação de proexadione cálcio no controle do desenvolvimento vegetativo de macieiras 'Imperial Gala'. Revista Brasileira de Fruticultura, Jaboticabal, v. 34, n. 4, p. 957-963, 2012b.

ILIAS, I.; RAJAPAKSE, N. Prohexadione-calcium affects growth and flowering of petunia and impatiens grown under photoselective films. Scientia Horticulturae, Amsterdam, v. 106, p. 190-202, 2005.

MAAS, F. Strategies to Control Tree Vigour and Optimise Fruit Production in 'Conference' Pears. Acta Horticulturae, The Hague, v. 800, p. 139146, 2008.

MEdjdouB, R.; VAL, J.; BLANCO, A. Prohexadione-Ca inhibits vegetative growth of 'Smoothee Golden Delicious' apple trees. Scientia Horticulturae, Amsterdam, v. 101, p. 243-253, 2004.

MILLER, S.S. Prohexadione-calcium controls vegetative shoot growth in apple. Journal of Tree Fruit Production, Binghamton, v. 31, p. 11-28, 2002.

PETRI, J.L.; LEITE, G.B.; HAWERROTH, F.J.; BASSO, C. Reduction of shoot growth and winter pruning in apple trees treated with prohexadione calcium. Acta Horticulturae, The Hague, v. 903, p. 873-878, 2011.

PRIVÉ, J.P.; CLINE, J.; FAVA, A. Influence of prohexadione calcium (Apogee ${ }^{\circledR}$ ) on shoot growth of non-bearing mature apple trees in two different growing regions. Canadian Journal of Plant Science, Ottawa, v. 86, p. 227-233, 2006.

PRIVÉ, J.P.; FAVA, E.; CLINE, J.E.; BYL, M. Preliminary results on the efficacy of apple trees fruit with the growth retardant prohexadionecalcium (Apogee) in the Eastern Canada. Acta Horticulturae, The Hague, v. 636, p. 137-144, 2004.

RADEMACHER, W. Prohexadione-Ca - A new plant bioregulator for use in apple production. In: ENCONTRO SOBRE FRUTICULTURA DE CLIMA TEMPERADO, 11., 2009, Fraiburgo. Anais... Florianópolis: Epagri, 2009. p. 1-10. 
RADEMACHER, W.; KOBER, R. Efficient use of prohexadione-Ca in pome fruits. European Journal of Horticultural Science, Stuttgart, v. 68, p. 101107, 2003.

RADEMACHER, W.; SPINELLI, F.; COSTA, G. Prohexadione-Ca: Modes of action of a multifunctional plant bioregulator for fruit trees. Acta Horticulturae, The Hague, v. 727, p. 97-106, 2006.
SANHUEZA, R.M.V.; PROTAS, J.F.S.; FREIRE, J.M. Manejo da macieira no sistema de produção integrada de frutas. Bento Gonçalves: Embrapa Uva e Vinho, 2006. 164p.

SAS INSTITUTE. Base SAS ${ }^{\circledR}$ procedures guide. Cary, 2004. 1861p. 